\title{
Advanced algorithms for radiographic material discrimination and inspection system design
}

\author{
Andrew J. Gilbert ${ }^{\mathrm{a}}$, Benjamin S. McDonald ${ }^{\mathrm{a}}$, Mark R. Deinert ${ }^{\mathrm{b}}$

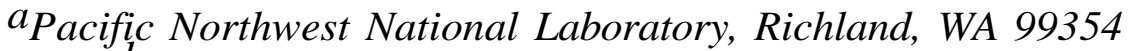 \\ ${ }^{b}$ Colorado School of Mines, Golden, CO 80401
}

\begin{abstract}
X-ray and neutron radiography are powerful tools for non-invasively inspecting the interior of objects. However, current methods are limited in their ability to differentiate materials when multiple materials are present, especially within large and complex objects. Past work has demonstrated that the spectral shift that X-ray beams undergo in traversing an object can be used to detect and quantify nuclear materials. The technique uses a spectrally sensitive detector and an inverse algorithm that varies the composition of the object until the X-ray spectrum predicted by $\mathrm{X}$-ray transport matches the one measured. Here we show that this approach can be adapted to multi-mode radiography, with energy integrating detectors, and that the Cramér-Rao lower bound can be used to choose an optimal set of inspection modes a priori. We consider multiendpoint X-ray radiography alone, or in combination with neutron radiography using deuteriumdeuterium (DD) or deuterium-tritium (DT) sources. We show that for an optimal mode choice, the algorithm can improve discrimination between high- $Z$ materials, specifically between tungsten and plutonium, and estimate plutonium mass within a simulated nuclear material storage system to within $1 \%$.
\end{abstract}

Corresponding authors. MR Deinert, Colorado School of Mines, email: mdeinert @ mines.edu; BS McDonald, Pacific Northwest National Laboratory, email: benjamin.mcdonald@pnnl.gov.

Introduction. Detecting the presence of nuclear material in an occluded configuration such as cargo remains problematic for current-generation X-ray radiography $[1,2]$. The methods used for doing this typically reconstruct an average attenuation coefficient for a given X-ray path length, and this makes it difficult to distinguish high-density materials from larger thicknesses of low-density ones. Computed tomography can help overcome this issue by giving a cross sectional view of an object that makes different material regions distinct. However, it adds to the system complexity and considerably slows scanning [3]. Image segmentation can also be used to determine locations of overlapping materials for better quantification [4], but it to can fail distinguish high-density materials from larger thicknesses of low-density ones. X-ray backscatter has been used to detect high atomicnumber materials, but shielding of the backscattered X-rays in the container can make detection and quantification difficult [5].

Another inherent problem with current generation X-ray radiography is that X-ray attenuation coefficients can be very similar over a wide range of energies for materials that are close in atomic number, Fig. 1a. Past work has shown that spectrally sensitive detectors, used in combination with an inverse algorithm, can improve the discrimination and quantification of nuclear materials [6]. However, the technique works best with low energy X-rays, and these 
can be easily shielded. Neutron radiography can help in situations such as these because their attenuation coefficients can show significant differences independent of atomic number, Fig. $1 \mathrm{~b}$, and because they can penetrate materials that X-rays cannot [7, 8]. Fast neutron radiography in particular has shown an increased capability to detect explosives compared to X-ray [9, 10]. A "dual-mode" system that uses both neutron and X-ray radiography has been shown to improve sensitivity to materials of similar atomic number in application to contraband in cargo, including special nuclear materials [11, 12]. A full-scale prototype dualmode system has been tested at the Brisbane International Airport and subsequently commercialized [13]. Systems such as these could also be important in the inspection of nuclear material storage containers for future treaty verification regimes. Here shielding is also an issue. Inspections of this type can be performed in some cases using neutron multiplicity counting to quantify the amount of plutonium in a container, but only if the isotopic composition is known [14].

Radiography systems that use both X-ray and neutron data are an active area of research [1113]. However, the optimal way in which to integrate multiple modes for material discrimination and quantification remains an open question. Here we show that an inverse algorithm with adaptive regularization can be used to optimize the use of multiple radiographic detection models for material discrimination and quantification. We consider multi-endpoint X-ray radiography alone, or in combination with neutron radiography using deuterium-deuterium (DD) or deuterium-tritium (DT) sources. We demonstrate the algorithms on a simulated inspection of an AT400R nuclear material storage container, Fig. 2. Because of the large set of possible system configurations from the two inspection types, we use the Cramér-Rao lower bound $[15,16]$ to pick a "best" inspection set from the available options before any optimizations are performed. It is shown that this approach is particularly useful for quickly designing an optimal inspection system for a given task.

Methods. The energy-dependent interaction of X-ray and neutron beams with an object comprised of $M$ materials can be described using [6]

$$
\Phi(\vec{t}, E)=\Phi_{0}(E) \exp \left[-\sum_{k=1}^{M} \mu_{k}(E) t_{k}\right]+\Phi_{\text {scatter }}(\vec{t}, E)
$$

Here, $\Phi$ [particles $\cdot \mathrm{cm}^{-1} \cdot \mathrm{keV}^{-1}$ ] is the X-ray or neutron energy-dependent fluence incident on the detector plane resulting from a source fluence $\Phi_{0}\left[\right.$ particles $\left.\cdot \mathrm{cm}^{-1} \cdot \mathrm{keV}^{-1}\right] ; \mu_{k}\left[\mathrm{~cm}^{-1}\right]$ is the energydependent linear attenuation coefficient for material $k$; $t_{k}$ is the thickness $[\mathrm{cm}]$ of material $k$ at the location of a given pixel in the image; and $\Phi_{\text {scatter }}\left[\right.$ particles $\cdot \mathrm{cm}^{-1} \cdot \mathrm{keV}^{-1}$ ] is the fluence from Xray or neutrons that are scattered (from the object or the surroundings) onto the image plane.

The corresponding detector response in a single image pixel can be represented by

$$
d(\vec{t})=c \int_{E} \Phi(\vec{t}, E) \varepsilon(E) P E d E
$$


Here, $c$ is a constant, $\varepsilon(E)$ is the detector efficiency [-], and $P$ [photons $\cdot \mathrm{keV}^{-1} \cdot$ particle ${ }^{-1}$ ] is the number of visible light photons produced in the scintillator per incident unit of particle energy $E[\mathrm{keV}]$. The constant $c$ [gray-levels-photon ${ }^{-1}$ ] assumes that the detector response is linear in energy, and we assume this for both X-ray neutron radiography systems.

The quantity of each of the $M$ materials at each pixel can be found for a combination of X-ray and neutron radiographs by minimizing, over $\vec{t}$ (a vector containing estimated material thicknesses at each image pixel), the objective function $Q$ given by

$$
\begin{aligned}
Q(\vec{t} ; \alpha, \beta)= & \frac{1}{2}\left\|\vec{N}_{(X)} \circ\left(\frac{\vec{d}_{(X)}(\vec{t})}{\vec{d}_{0(X)}}-\frac{\vec{d}_{o b s(X)}}{\vec{d}_{0, o b s(X)}}\right)\right\|\left\|^{2}+\frac{1}{2}\right\| \vec{N}_{(n)} \circ\left(\frac{\vec{d}_{(n)}(\vec{t})}{\vec{d}_{0(n)}}-\frac{\vec{d}_{o b s(n)}}{\vec{d}_{0, o b s(n)}}\right) \|^{2} . \\
& +\alpha \sum \sqrt{\left(\mathbf{D}_{x} \vec{t}\right)^{2}+\left(\mathbf{D}_{y} \vec{t}\right)^{2}+\beta}
\end{aligned}
$$

Here $(X)$ and $(n)$ denote X-ray and neutron, and $\vec{d}_{o b s}$ is a vector containing the observed signals at each pixel for each inspection. Further, o denotes a component-wise (Hadamard) multiplication, where $\vec{x}^{2}=\vec{x} \circ \vec{x}$, and $\vec{x} / \vec{y}$ denotes component-wise division. The data terms are scaled, pixel-by-pixel, by an unattenuated image $d_{0}$, which divides out variation in the detector response and source intensity across the image plane. The third term in Eq. (3) is the total variation regularization [17] and $\alpha$ varies its strength. This form of regularization penalizes the objective function for solutions that have large variation in the material estimates from one pixel to the next. The term includes matrices $\mathbf{D}_{\mathbf{y}}$ and $\mathbf{D}_{\mathbf{x}}$, which calculate the backwards finite difference of the estimated material thicknesses in the row $y$ and column $x$ dimensions [18]. The term $\beta$ ensures that Eq. (3) is differentiable with respect to $\vec{t}$, necessary for the use of Newton-type solvers used in this work. It is set to $\beta=1$ in this study. The misfit terms are normalized by the vector $\vec{N}$, which is chosen to prevent the largecount data in $\vec{d}$ from overweighting the norm as well as to simplify the adaptive $\alpha$ selection method. As in previous work [6], the normalization is chosen so that normalized data is standard normally distributed, here $\vec{N}_{(X, n)}=\sqrt{\vec{d}_{0,(X, n)}} / \sqrt{\vec{d}_{(X, n)}\left(\vec{t}_{\ell-1}\right)}$, where $\ell$ 1denotes the thickness vector from the previous optimization iteration.

The objective function in Equation (3) is nonlinear; we use a Gauss-Newton method with linesearch to solve it [17]. This type of optimization is iterative, approximating the objective function as a quadratic at each step and minimizing that approximation. This requires calculation of the first and second derivatives of the objective function with respect to $\vec{t}$ at each iteration. For the Gauss-Newton method, an analytical first derivative (the gradient) is used, but the second derivative matrix (the Hessian) is approximated with the Jacobian matrix, which reduces computation time and was found to perform comparably to when the full Hessian is calculated. A non-negativity constraint is put on the solution by using the projected-gradient, reduced-Hessian method [17]. The iterations are stopped when the maximum 
change in $\vec{t}$ is less than $10^{-4}$, which is found to be sufficient for the object geometry in this work. All codes are written and implemented in Matlab (Mathworks, Natick, MA); a vast majority of the code was written by the authors.

It has previously been shown that $\alpha$ can be found adaptively for this type of inverse problem [6] and the same methodology is applied here, where the $\alpha$ parameter is selected using the unbiased predictive risk estimate method [17]. Finding this term automatically in an inspection is important to ensure adequate results and minimize potential operator error and interfacing requirements. The typical computational time to process each image on a single CPU, with the adaptive $\alpha$, is on the order of 10 minutes, which could be reduced with parallelization and code optimization.

Optimal selection of inspection modes. Inspection parameters that can be varied in the design of a radiography system include the endpoint energy of the X-ray spectra, the reaction type of the neutron generator, and the detector efficiencies. Here we consider nine different Bremsstrahlung X-ray source spectra (1-9 MVp) as well as neutrons generated from DD and DT reactions. This gives a set of 11 parameter options from which a dualmode system can be chosen. The current study is limited to the detection and measurement of four materials; thus we seek the best combination of four inspection energies/types from this set for this study. We use the Cramér-Rao lower bound to estimate how each of these affects the noise in the estimates of the material inside an AT400R. Roessl and Herrman showed that minimization of noise in this way can be used as a design criterion for optimizing a radiography system $[15,16]$.

The Cramér-Rao lower bound uses the Fischer information matrix $F$ to approximate the variance in the material estimates. Assuming Poisson statistics, the entries in $F$ are given by [15]

$$
F_{p, q}=\sum_{l} \frac{1}{\sigma_{l}^{2}} \frac{\partial d_{l}(\vec{t})}{\partial t_{p}} \frac{\partial d_{l}(\vec{t})}{\partial t_{q}}
$$

where $d_{l}$ is the data model for a given inspection and $\sigma^{2}=d_{l}$. The partial derivative with respect to a single material thickness $t_{p}$ is given by

$$
\frac{\partial d_{l}(\vec{t})}{\partial t_{p}}=-c \int_{E} \mu_{p}(E) \varepsilon(E) P E \Phi_{0, l}(E) \exp \left[-\sum_{k=1}^{M} \mu_{k}(E) t_{k}\right] d E,
$$

and equivalently for $t_{q}$. For a given material estimate from an unbiased estimator, such as that obtained from a least-squares minimization problem, the Cramér-Rao lower bound on the material estimate variance is given by

$$
{ }_{t_{p}}^{2} \quad F_{p p}{ }^{1}, \quad p=1, \square, M .
$$


Here $F^{-1}$ is the inverse of the Fischer information matrix given in Eq. (6), where the " $p p$ " subscript denotes the diagonal of $F$ at the $p$ 'th location. Similar to [15], the figure of merit used here is the minimum material signal-to-noise ratio (SNR), which is related to the variance as

$$
S N R_{t_{p}}=\frac{t_{p}}{t_{p}} .
$$

To calculate the Cramér-Rao lower bound, material thicknesses must be chosen beforehand. Here we use two material sets. The first is $\{\mathrm{Pu}$, steel, polypropylene $\}$ with thicknesses $(4 \mathrm{~cm}$, $2.5 \mathrm{~cm}$, and $28 \mathrm{~cm}$ ), representative of those that would be seen in the AT400R [19]. The second set is a four-material-set of $\{\mathrm{Pu}$, tungsten $(\mathrm{W})$, steel, and polypropylene $\}$, with thicknesses $\{4$ $\mathrm{cm}, 0.1 \mathrm{~cm}, 2.5 \mathrm{~cm}, 28 \mathrm{~cm}$, where tungsten is included as a material that might be substituted for plutonium if diversion of nuclear material were being attempted. It was found that the value of the Cramér-Rao lower bound doesn't depend strongly on the material thicknesses used.

$X$-ray and neutron source models. The X-ray source spectra are modeled using the Monte Carlo simulation package MCNP5 [20]. Bremsstrahlung sources are typically used for high-energy Xray scans [4] and are assumed here. The X-ray source spectra are generated in MCNP by tracking monoenergetic electrons impinging on a tungsten target with a $30^{\circ}$ takeoff angle and tallying the resulting Bremsstrahlung X-rays coming out of the target towards the image plane. This source spectrum is filtered with $2 \mathrm{~cm}$ of lead to increase the average energy of the X-ray spectrum. X-ray source spectra of maximum energies from 1 to $9 \mathrm{MeV}$, in 1-MeV steps, are produced, as shown in Fig. 3a. Neutron spectra from DD and DT reactions are taken from [21], and are shown in Fig. 3b. The X-ray and neutron spectra are binned into groups at 5-keV intervals.

Detector model. The modeled X-ray detector is a $2 \mathrm{~cm}$ thick cadmium tungstate scintillator. The detector integrates over the flux incident at each pixel, and the detector response is modeled as an efficiency curve where an interaction of any kind in the detector medium results in a detection. It has previously been shown that a detector model based on efficiency is a reasonable approximation (e.g., 22).

The fast-neutron detector model is based on an associated particle imager such as those used in the Nuclear Materials Identification System [23] and the Advanced Portable Neutron Imaging System [24]. The radiography detector simulated in our work is based on the pixelized detector used in the latter with a pixel size of $1 \times 1 \times 5 \mathrm{~cm}^{3}$ of polyvinyl toluene (PVT). The neutron detector assumed here is quite thick, which would result in reduced spatial resolution. However, for the inspection of large dense objects, greater neutron efficiency becomes important for observing the small number of transmission neutrons 
coming through the thickest regions of the object.

Regarding the neutron detector efficiency, since fast neutron detectors based on PVT rely on the recoil reaction with hydrogen as the mechanism for detection, any other elements in the detector have a deleterious effect. In the case of PVT, carbon is another major component in the scintillator. The neutron detection efficiency is approximated here as [25]

$$
{ }_{n}=\frac{H}{{ }_{H}+{ }_{C}}\left\{1 \exp \left({ }_{H}+{ }_{C}\right) z\right\}
$$

Here $\Sigma_{H}$ and $\Sigma_{C}\left[\mathrm{~cm}^{-1}\right]$ are the linear neutron attenuation coefficients and $z[\mathrm{~cm}]$ is the detector thickness. This equation ignores the effect of multiple neutron scatters in the detector medium and multiple-pixel interactions.

More complicated physics such as partial energy deposition, edge effects, and the effect of image spatial resolution are ignored. In addition, though organic plastic scintillator neutron detectors such as PVT have a nonlinear $\left(\alpha E^{3 / 2}\right)$ response in energy [25], this effect within the energy bands of the DD and DT source spectra (Fig. 3b) is small and is, therefore, ignored here.

Radiation transport model. The Monte Carlo transport code, MCNP5 [20], is used for simulating radiation transport from the source to the image plane flux. For the MCNP radiation transport model, the source spectra shown in Fig. 3 are used as the incident flux. The AT400R, Fig. 2, is surrounded by dry air with a source-to-object distance of 2 $\mathrm{m}$ and an object-to-detector distance of $1 \mathrm{~m}$.

A radiography tally for both X-rays and neutrons is used to determine the respective flux at the imaging plane from each source type. The radiography tally is $1 \mathrm{~m} \times 1 \mathrm{~m}$ in total size with a pixel array of $100 \times 100 ; 1 \mathrm{~cm}$ pixel pitch. Note, the simulations assume that the X-ray and neutron radiographs are fully registered. Though it is expected that the neutron radiograph would have lower spatial resolution than the $\mathrm{X}$-ray radiograph, this could be remedied in practice by subsampling the X-ray radiograph. The MCNP model can separately tally the directly transmitted flux as well as that resulting from scatter off of the object or the surroundings. The simulated image data for the $8 \mathrm{MVp}$ Bremsstrahlung $\mathrm{X}$-ray source and the DT neutron source are shown in Fig. 4.

The flux tallies at the image plane for X-rays are binned into 50-keV wide energy groups up to the maximum spectrum energy. The neutron flux tallies are binned into $100-\mathrm{keV}$ wide energy groups from 1.8 to $3 \mathrm{MeV}$ for the DD image and from 12.4 to $15.7 \mathrm{MeV}$ for the DT image. The neutron tallies also have a low-energy bin with a low edge at $1 \mathrm{keV}$ to capture the low-energy, scattered neutrons. Bin sizes were selected to maintain good accuracy in the modeled flux and a reasonable computation time to complete the 
simulations.

Scatter flux model. The simulated flux is split into uncollided and scattered components in the MCNP tallies. This also gives an accurate representation of the profile of the scattered flux for such an inspection. The scattered flux component for the 6-MVp X-ray radiograph is shown in Fig. 5 as a fraction of the unattenuated counts in each pixel. Notice that the scattered flux makes up a considerable portion of the image - here as much as $5 \%$ of the maximum signal in the image. This is another source of noise in the image data and effectively reduces the dynamic range of the inspection system. A degree of scatter rejection for either the X-ray or neutron image is necessary and assumed for accurate material discrimination. How the results vary with varying levels of scatter allowed on the image plane is explored in the results section. Here, it is assumed that some information about the scattered flux component is known. To simulate this, a two-dimensional, secondorder polynomial is fit to the scatter component, which is supplied to the inverse algorithm. This approximation is accurate to within $3-5 \%$ of the actual, simulated scatter component.

Noise model. The noise in the data is assumed to be dominated by counting statistics, which is Poisson distributed. After the image data has been modeled, it is scaled so that the maximum number of counts in a single background (unattenuated) pixel is $10^{6}$ for both the X-ray and neutron radiographs and noise is added by drawing independent Poisson random numbers for each pixel assuming the modeled pixel value is the mean. The same scaling is used for the X-ray and neutron radiographs. For the neutron radiographs, this scaling is consistent with a $\sim 5$ minute acquisition with a $2 \times 10^{10} \mathrm{n} / \mathrm{s}$ DT neutron generator or a $\sim 20$ minute acquisition with a $4 \times 10^{9} \mathrm{n} / \mathrm{s}$ DD neutron generator placed at $2 \mathrm{~m}$ from a detector of $10 \%$ efficiency. Since the object being inspected is large and of high-density, this results in a wide range of SNR in the image data, from about $10^{3}$ in the background pixels to 50 in the most attenuated regions of the $2 \mathrm{MVp}$ X-ray image. In an operational environment, other sources of noise would likely be present, such as instability in the X-ray and neutron sources, instability in the detector system, and nonlinearities in the neutron detector response. These can be mitigated with a well-characterized inspection system and regular calibration, and would have to be taken into account in selecting equipment for such an inspection system, where stability is especially important.

Calculation of error. For a measure of goodness-of-fit, the root mean square error is used here, comparing the estimated material thicknesses from the algorithm to those actually present. Two measures of the root mean square error (RMSE) are calculated, the RMSE $\mathrm{Pu}$ which only calculates the error where plutonium is located, and the RMSE $E_{\text {not } P u}$, which calculates the error where plutonium is not located. These measures indicate accuracy in plutonium quantification and erroneous quantification, respectively. Though these measures are used here to quantify errors, in an actual inspection they could not be directly quantified since the true location of the plutonium would likely be unknown. The plutonium mass error is calculated by comparing the total plutonium mass estimated from the algorithm to that actually present. This error is calculated only where plutonium is actually located, so it does not capture any plutonium estimated where it is not actually present. 
Results. Radiographs were simulated for all possible combinations of X-ray endpoint energies and DD and DT neutron sources. From these possible inspections, all possible sets of four were chosen, giving a total of 330 unique inspection sets, and the optimization algorithm was run without regularization for each. The panels in Fig. 6 show the median values of the Cramér-Rao SNR and the RMSE for all sets of each inspection type (i.e., results from the $\{2,4,6,8 \mathrm{MVp}\}$ and $\{3,4,5,6 \mathrm{MVp}\}$ inspection sets are both included in the median X-ray value shown in the panels). Results using a three-material-set $\{\mathrm{Pu}$, steel, poly-pro $\}$ are shown in panel $6 \mathrm{a}$, and results for a four-material-set $\{\mathrm{Pu}$, tungsten $(\mathrm{W})$, steel, poly-pro $\}$ are shown in panel 6b. These results show that the RMSE is reduced with the addition of neutron radiography data and, importantly, a correlation between a decrease in the RMSE and an increase in the SNR is indicated. This effect is pronounced when tungsten is added to the set of materials, Fig. 6b. The results also show that there is varied solution precision depending on the set of inspections chosen, as others have shown [15]. Though the maximum SNR doesn't necessarily correlate to the minimum plutonium RMSE, the Cramér-Rao SNR is shown here to be an effective and efficient measure, especially for designing inspection systems that may have a large variety of inspected objects and inspection parameters (i.e., radiation type, source strength, detector type, imaging geometry, etc.).

Using the SNR as a measure of quality, parameter sets were chosen that had the highest SNRs within their inspection type and their performances compared. The chosen inspection sets are summarized in Table 1. Table 2 gives the results from the algorithm using the inspection sets in Table 1 and a three-material-set $\{\mathrm{Pu}$, steel, poly-pro\}. Estimates for the plutonium mass (calculated in the pixels where the plutonium actually exists) are within $1 \%$ in all cases. In order to separate the performance of the Cramér-Rao lower bound from the adaptive $\alpha$ selection, the value for $\alpha$ is chosen optimally here based on the known material composition. The RMSE for the dual-mode inspection is lower than that with the X-ray only inspection but this particular problem indicates that X-ray inspection alone may be sufficient for accurate $(<1 \%$ error) noninvasive estimations of material mass with the use of inverse problem algorithms. However, the plutonium mass estimated when a plutonium sphere is not present (the null case) is significantly lower using a dual-mode inspection $-0.74 \pm 0.47$ grams $(<0.1 \%)$, compared to that with $X$-ray alone, 23.5 \pm 3.3 grams $(1.2 \%)$. Of course, the inspection set chosen will also depend on the task.

While for certain problems X-ray inspection alone may be sufficient to quantify materials such as plutonium, an intelligent spoof attempt could potentially fool even a multi-energy X-ray scan. An example of this is if one (or both) of the plutonium spheres in an AT400R were replaced with another high-density, high-atomic number material, such as tungsten. Figure 7 shows a simulated 5-MVp X-ray image ( $\log _{10}$-transformed) of this situation, illustrating how the similarity of these two materials could result in an undetected material diversion. In this situation, complementary sources of data, such as neutron radiography, could be especially useful. Material estimates made using Eq. (3) for this situation are given in Fig. 8 for both X-ray and dual-mode inspections. Scatter is not included in the image simulations here. Figure 8 shows results when a three-material-set of $\{\mathrm{Pu}$, steel, poly-pro $\}$ is 
used. These results show that using X-ray radiographs alone, the algorithm gives the expected plutonium quantity where, in actuality, the tungsten sphere is located (Fig. 8-left). In contrast, the difference between the plutonium and tungsten can be seen much more clearly when neutron radiography data are used (Fig. 8-right). The plutonium mass errors for the X-ray only and dual-mode results are $103 \pm 1 \%$ and $81 \pm 1 \%$, respectively, indicating that the $\mathrm{X}$-ray estimation is finding the full profile of the tungsten sphere as if it were plutonium while there is clearly a qualitative difference in the dual-model estimation.

Figure 9 shows the results when a four-material-set is used, with tungsten (W) being the additional material. With X-ray alone, the algorithm clearly cannot distinguish between plutonium and tungsten, and the polypropylene accuracy is also reduced. Here, the plutonium mass errors for the X-ray-only and dual-mode results are $51 \pm 20 \%$ and $4.4 \pm 2.7 \%$, respectively, indicating significantly better performance by the dual-mode scan. The dualmode results show reduced accuracy compared to the three-material-set (Fig. 8), but indicate significant utility in discriminating between two materials of similar atomic number and density, compared to using only X-ray. If needed, the results could be used qualitatively, as a means of detecting materials rather than accurately quantifying them. Also, if a different material is suspected, such as uranium instead of tungsten replacing the plutonium, multiple runs of the algorithm could be made in parallel with various material sets to determine the most likely material.

Scattered flux at the image plane lowers the SNR and has a negative effect on the precision of the material estimates. The effect is greatest in regions where the flux is most attenuated. Table 3 summarizes how the plutonium mass estimates vary with the addition of different amounts of scatter in the inspection model. As expected, the error in plutonium estimates is increased with the addition of scattered flux in the image data, even when a good estimate of the scatter term is included in the physics model in the inverse problem. The effect of X-ray scattering can be reduced using anti-scatter grids and by increasing object-to-detector distance. Also, the associated particle neutron imagers are effective at rejecting scattered flux by correlating in time and space the neutron at the image plane with the associated alpha produced in the DD or DT reaction [23].

Conclusions. We have shown that an inverse-problem algorithm with adaptive regularization can be used to optimize the integration of X-ray and neutron radiographic data for inspection of nuclear-material storage containers. Using simulated X-ray and neutron radiographs, we found that the accuracy of material estimates increased when X-ray and neutron radiography are used together, compared to inspection with X-rays only. A previously developed inverse algorithm was adapted for use in these simulations and was found to perform well in this application. The plutonium mass, calculated in the pixels where it actually exists, was consistently estimated to within $1 \%$ of its actual value. Importantly, the utility of using neutron radiography as complementary to X-ray radiography was shown in the increased material accuracy determined when the nuclear material was replaced with another high-atomic-number, high-density material. Finally, the Cramér-Rao lower bound was used to pick an optimal inspection set a priori from the available X-ray and neutron inspection energies, which is useful for designing inspection systems for targeted applications. 
Acknowledgements. This research was sponsored by the Office of Nonproliferation Research and Development, National Nuclear Security Administration. A. J. Gilbert was supported by the Next Generation Safeguards Initiative, Office of Nonproliferation and Arms Control. A portion of the research was performed using PNNL Institutional Computing at Pacific Northwest National Laboratory. Pacific Northwest National Laboratory is operated for the U.S. Department of Energy by Battelle under Contract DE-AC05-76RL01830. PNNL-SA-113690.

\section{References}

[1] G. Chen, G. Bennett, and D. Perticone, "Dual-energy X-ray radiography for automatic highZ material detection," Nuclear Instruments \& Methods in Physics Research Section B-Beam Interactions with Materials and Atoms, vol. 261, no. 1-2, pp. 356-359, Aug, 2007.

[2] Y. Liu, B. D. Sowerby, and J. R. Tickner, "Comparison of neutron and high-energy X-ray dual-beam radiography for air cargo inspection," Applied Radiation and Isotopes, vol. 66, no. 4, pp. 463-473, Apr, 2008.

[3] Y. Xing, L. Zhang, X. Duan, J. Cheng, and Z. Chen, "A Reconstruction Method for Dual High-Energy CT With MeV X-Rays," IEEE Transactions on Nuclear Science, vol. 58, pp. 537546, 2011.

[4] S. Ogorodnikov, V. Petrunin, Processing of interlaced images in 4-10 MeV dual energy customs system for material recognition, Physical Review Special TopicsAccelerators and Beams, 5 (2002).

[5] W. Bertozzi, S. E. Korbly, R. J. Ledoux, W. Park, Nuclear resonance fluorescence and effective $\mathrm{Z}$ determination applied to detection and imaging of special nuclear material, explosives, toxic substances and contraband, Nuclear Instruments and Methods in Physics Research Section B: Beam Interactions with Materials and Atoms, 261 (2007) 331-336.

[6] A. J. Gilbert, B. S. McDonald, S. M. Robinson, K. D. Jarman, T. A. White, M. R. Deinert, Non-invasive material discrimination using spectral X-ray radiography, Journal of Applied Physics, 115 (2014).

[7] R. Runkle, T. White, E. Miller, J. Caggiano, and B. Collins, "Photon and neutron interrogation techniques for chemical explosives detection in air cargo: A critical review," Nuclear Instruments and Methods in Physics Research Section A: Accelerators, Spectrometers, Detectors and Associated Equipment, vol. 603, no. 3, pp. 510-528, 2009.

[8] ENDF/B-VII Evaluated data, 1997.

[9] J. C. Overley, M. S. Chmelik, R. J. Rasmussen, R. M. S. Schofield, and H. W. Lefevre, "Explosives detection through fast-neutron time-of-flight attenuation measurements," Nuclear Instruments and Methods in Physics Research Section B: Beam Interactions with Materials and 
Atoms, vol. 99, no. 1-4, pp. 728-732, 1995.

[10] J. C. Overley, M. S. Chmelik, R. J. Rasmussen, R. M. S. Schofield, G. E. Sieger, and H. W. Lefevre, "Explosives detection via fast neutron transmission spectroscopy," Nuclear Instruments and Methods in Physics Research Section B: Beam Interactions with Materials and Atoms, vol. 251, no. 2, pp. 470-478, 2006.

[11] J. E. Eberhardt, S. Rainey, R. J. Stevens, B. D. Sowerby, J. R. Tickner, Fast neutron radiography scanner for the detection of contraband in air cargo containers, Applied Radiation and Isotopes 63 (2005) 179-188.

[12] S. Van Liew, "X-ray and neutron interrogation of air cargo for mobile applications," Nuclear Instruments and Methods in Physics Research Section A: Accelerators, Spectrometers, Detectors and Associated Equipment, vol. 784, pp. 417-422, 2015.

[13] N. G. Cutmore, L. Yi, and J. R. Tickner, "Development and commercialization of a fastneutron/x-ray Cargo Scanner." In IEEE International Conference on Technologies for Homeland Security (HST), 2010.

[14] R. Kouzes and B. Geelhood, "Composite Signatures of Nuclear and Non-Nuclear Technologies for Weapons Material and Component Measurement," Pacific Northwest National Laboratory, Richland, WA, 2002 (PNNL-13861).

[15] E. Roessl, C. Herrmann, Cramér-rao lower bound of basis image noise in multipleenergy x-ray imaging, Physics in Medicine and Biology, 54 (2009) 1307.

[16] A. S. Wang, N. J. Pelc, Sufficient statistics as a generalization of binning in spectral xray imaging, IEEE Transactions on Medical Imaging, 30 (2011) 84-93.

[17] C. R. Vogel, Computational Methods for Inverse Problems, SIAM, Philadelphia, 2002.

[18] J. M. Bardsley, and A. Luttman, "Total variation-penalized Poisson likelihood estimation for ill-posed problems," Advances in Computational Mathematics, vol. 31, no. 1-3, pp. 35-59, 2009.

[19] J. E. Tanner, S. D. Miller, M. M. Conrady, and J. Benz, "Autoradiography using OSL for monitoring warhead dismantlement," in Proceedings from the 51 st Annual INMM Meeting, Baltimore, Maryland, 2010.

[20] MCNP X-5 Monte Carlo Team, A General Purpose Monte Carlo N-Particle Transport Code, Version 5, LA UR 03 1987, Radiation Safety Information Computational Center, Los Alamos, NM, 2003.

[21] T. P. Lou, Compact D-D/D-T neutron generators and their applications, Thesis, 2003.

[22] A. J. Gilbert, B. S. McDonald, M. R. Deinert, Noninvasive material discrimination of 
high-atomic-numbered materials with multi-spectral x-ray radiography, 2016. In submission.

[23] P. A. Hausladen, P. R. Bingham, J. S. Neal, J. A. Mullens, J. T. Mihalczo, Portable fast-neutron radiography with the nuclear materials identification system for fissile material transfers, Nuclear Instruments and Methods in Physics Research Section B: Beam Interactions with Materials and Atoms, 261 (2007) 387-390.

[24] M. A. Laubach, J. P. Hayward, X. Zhang, and J. W. Cates, "Timing and position response of a block detector for fast neutron time-of-flight imaging," Nuclear Instruments and Methods in Physics Research Section A: Accelerators, Spectrometers, Detectors and Associated Equipment, vol. 763, pp. 495-501, 2014.

[25] G. F. Knoll, Radiation Detection and Measurement, 3rd ed., John Wiley \& Sons, Inc., 2000.

[26] M. Collins, A. Belian, Mayak AT-400R inspection MCNP model, Los Alamos National Laboratory, 2000. 


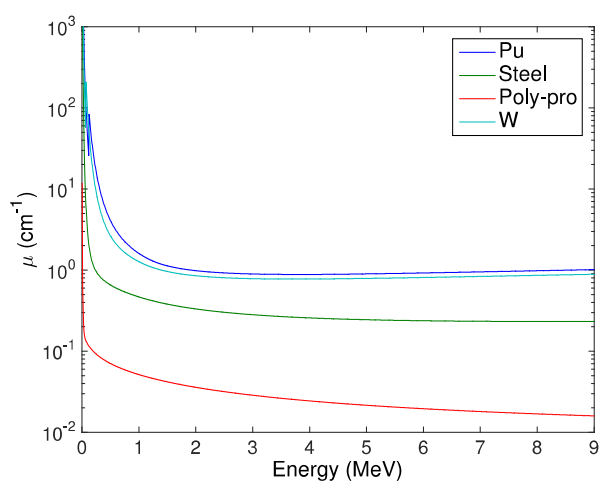

(a)

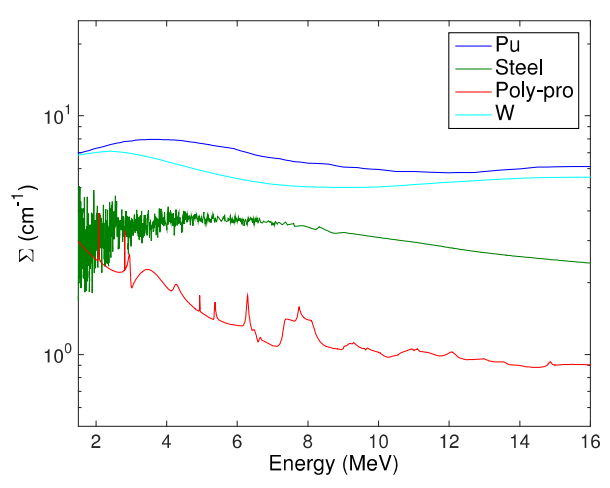

(b)

Figure 1. X-ray and neutron linear attenuation coefficients. (a) X-ray linear attenuation coefficients with respect to energy for plutonium $(\mathrm{Pu})$, tungsten $(\mathrm{W})$, steel, and polypropylene. (b) Neutron linear attenuation coefficients as a function of energy for the same materials. For X-rays there is very little difference between the linear attenuation coefficients of plutonium and tungsten. By contrast, there is a great deal of variation in the neutron attenuation coefficients. The plutonium linear attenuation coefficients assumed $\alpha-\mathrm{Pu}$, with isotopics of $94 \% \mathrm{Pu}-239$ and $6 \% \mathrm{Pu}-240$ and density of $19.1 \mathrm{~g} / \mathrm{cm}^{3}$. Linear attenuation coefficients for both X-ray and neutrons were obtained from the ENDF libraries [7]. 

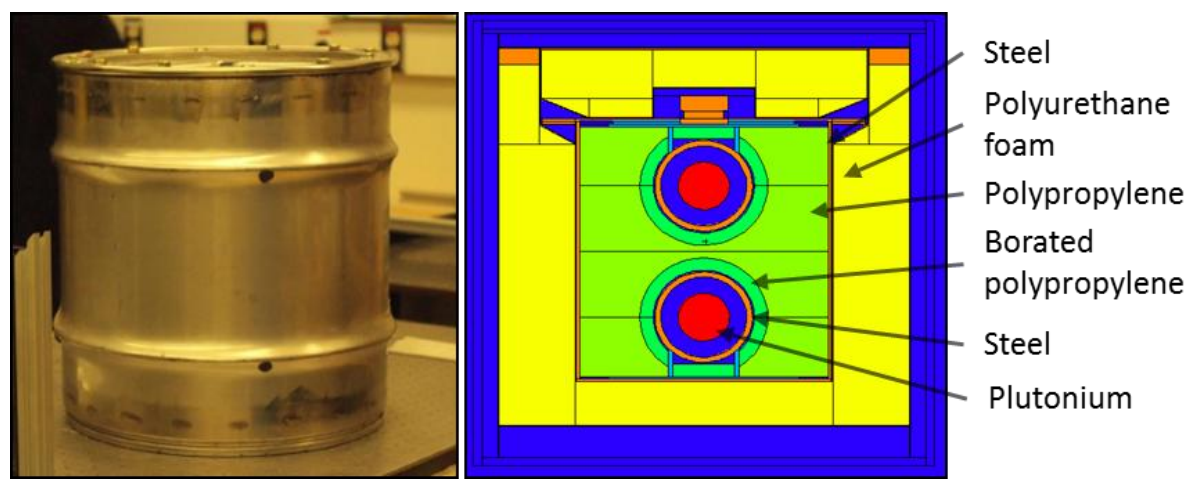

Figure 2. AT400R nuclear materials storage container. A picture (left) and cross-sectional schematic (right) of the AT400R object, which is the inspected item in this study. It is assumed to contain two $\sim 2 \mathrm{~kg}$ plutonium spheres of $\sim 5.8 \mathrm{~cm}$ diameter shielded with stainless steel (SS-304) and borated polypropylene. These geometries are placed in a steel can of $\sim 30 \mathrm{~cm}$ diameter which is filled with lowdensity polypropylene. 


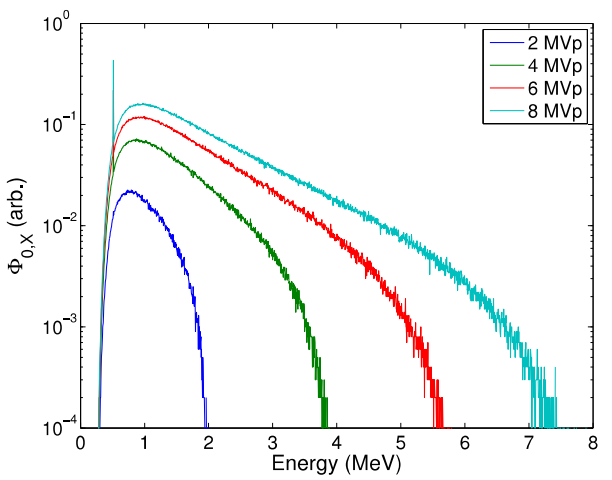

(a)

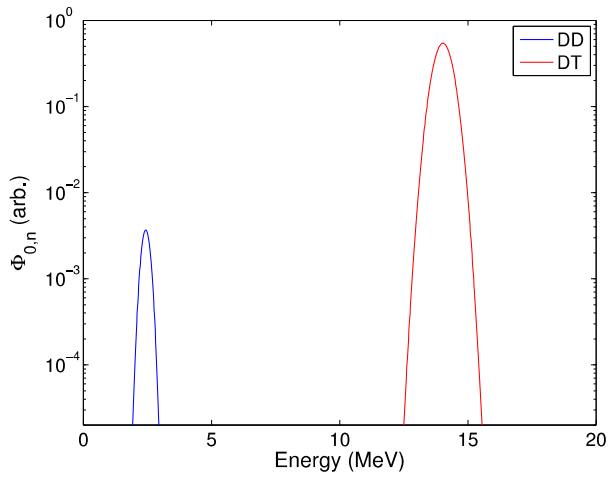

(b)

Figure 3. X-ray and neutron energy spectra. (a) X-ray spectra generated using MCNP5 for four different endpoint energies. (b) Neutron spectra for DD and DT fusion reactions. 


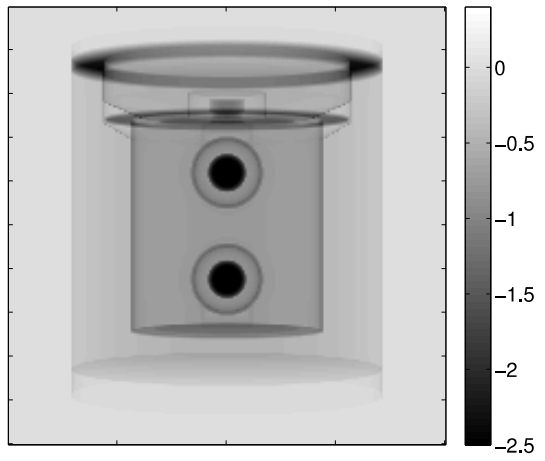

(a)

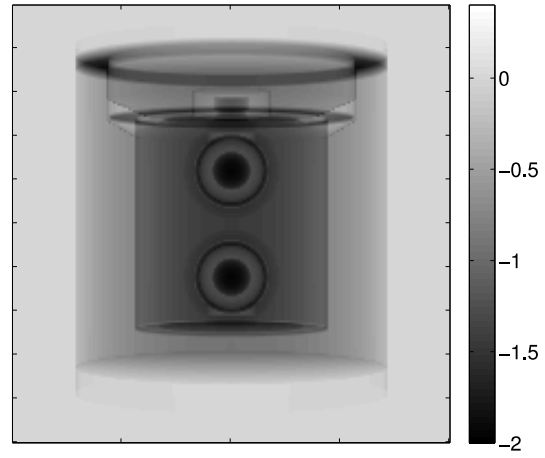

(b)

Figure 4. Simulated $X$-ray and neutron radiography images of the AT400R. (a) A synthetic X-ray image from the $8 \mathrm{MVp}$ Bremsstrahlung source. (b) A synthetic neutron radiograph from a DT source. Image data is $\log _{10}$-transformed so that the AT400R structure can be seen in the heavily attenuated regions of the image. Note the different contrast between the two images, where the neutron radiograph shows greater attenuation from hydrogenous materials such as polypropylene compared to the X-ray radiograph. 


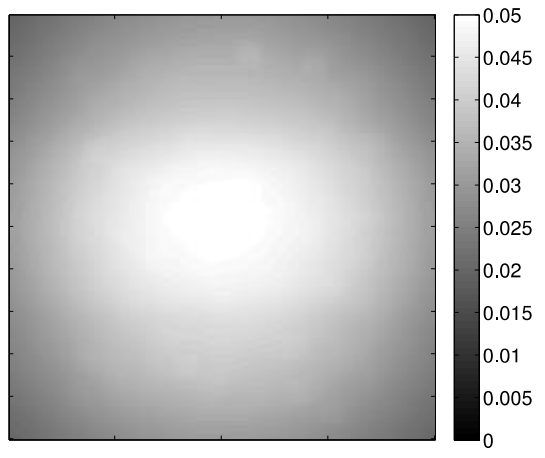

(a)

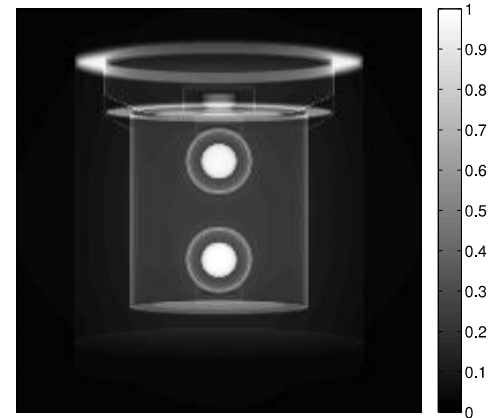

(b)

Figure 5. The scattered flux component of the 6 MVp X-ray image, shown as a fraction of the unattenuated signal in the image data. (a) The scattered $X$-ray flux is shown and is a considerable component of the image intensity, here as much as $5 \%$ in the center of the image which is also the location with the lowest transmission through the object. (b) The fractional scatter per pixel, here the ratio of the scattered pixel data to the total pixel data, shows where the scatter component will be most important, behind the highly attenuated regions of the image. 


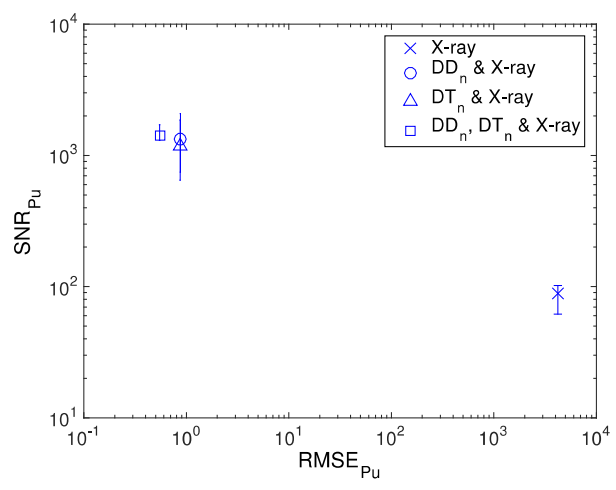

(a)

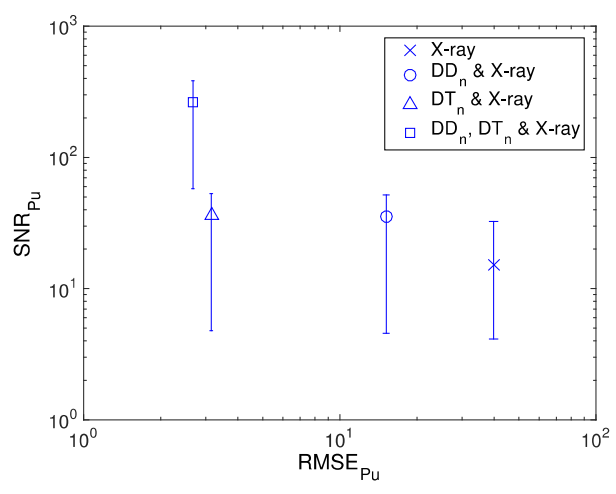

(b)

Figure 6: The relationship between the median plutonium root mean square error (RMSE) and the plutonium Cramér-Rao SNR. (a) The results when the three-material-set $\{\mathrm{Pu}$, steel, polypropylene $\}$ is used. (b) The results with the fourmaterial-set $\{\mathrm{Pu}, \mathrm{W}$, steel, polypropylene $\}$. A correlation of decreased RMSE with increased SNR is suggested by looking at the median values of all the inspection sets given to the algorithm of each type: X-ray only; DD neutron and X-ray; DT neutron and X-ray; and DD, DT neutron and X-ray. The error bars indicate the upper and lower quartiles. The median is used instead of the mean since it is not skewed by large values in the RMSE, due to the instability of the problem solutions. (In fact, the quartiles on the RMSE are not shown because of the large variation in RMSE among the sets of each inspection type.) The large variation in the SNR in (b) indicates that some inspections will perform considerably better than others. 


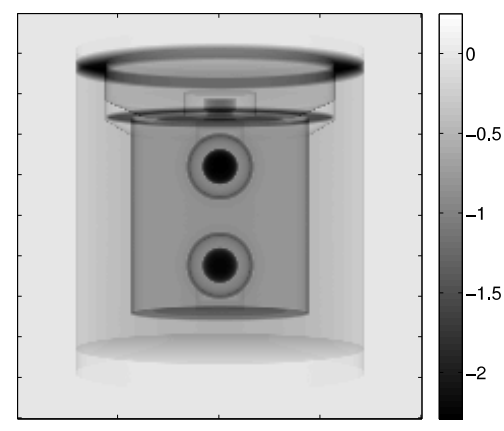

(a)

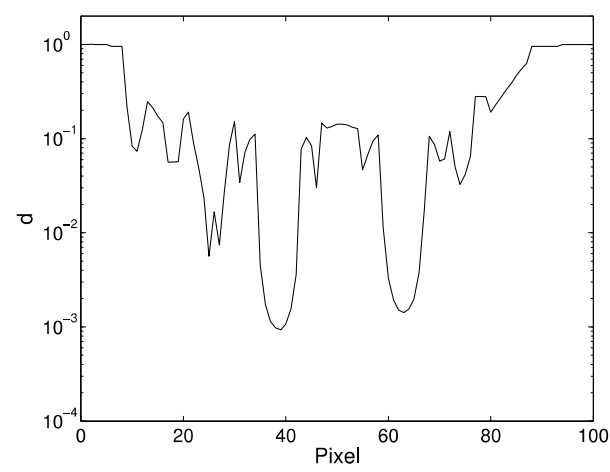

(b)

Figure 7. Single energy radiograph of a nuclear material container with both plutonium and tungsten. (a) A 5-MVp X-ray radiograph ( $\log _{10}$-transformed) with a tungsten sphere replacing the bottom plutonium sphere. Due to the similarity of tungsten and plutonium in atomic number and density, they are practically indistinguishable in the X-ray data alone. (b) A line scan line of the radiograph that passes through the two spheres. From X-ray transmission alone, the plutonium and tungsten spheres look very similar. 

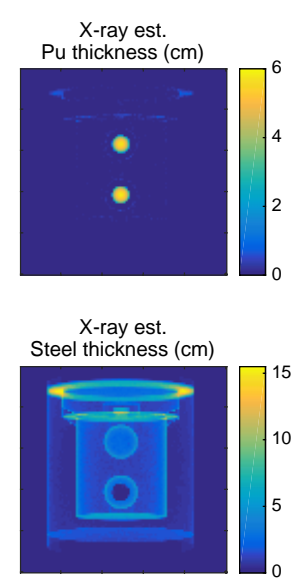

X-ray est.

Poly-pro thickness (cm)

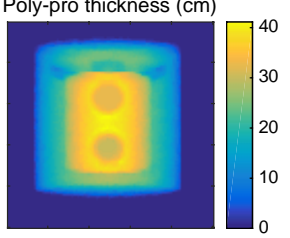

X-ray \& neutron est.

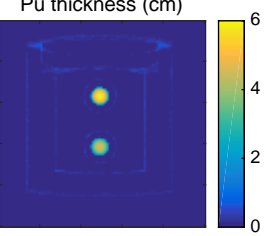

X-ray \& neutron est. Steel thickness $(\mathrm{cm})$

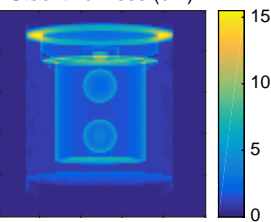

X-ray \& neutron est. Poly-pro thickness (cm)

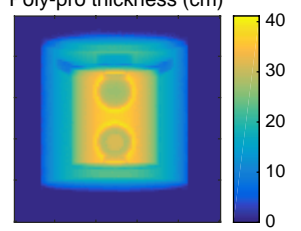

Figure 8. Material estimates of a nuclear material container with both plutonium and tungsten based on scans of only $\mathrm{X}$-ray radiography or $\mathrm{X}$-ray and neutron radiography. As an example of a possible material diversion scenario, the bottom plutonium sphere is replaced with a tungsten sphere of the same size. Three materials are sought $\{\mathrm{Pu}$, steel, poly-pro\}. (Left) Material estimates made using X-rays alone. (Right) Material estimates made using X-ray as well as the DT-source neutron radiograph. 

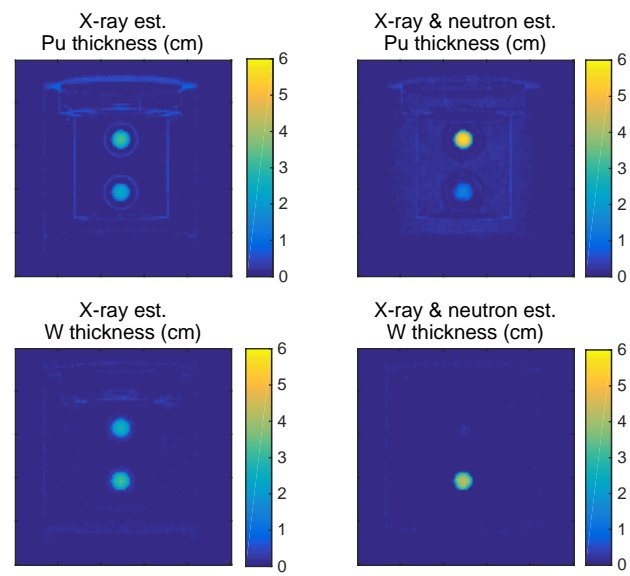

X-ray \& neutron est. W thickness $(\mathrm{cm})$

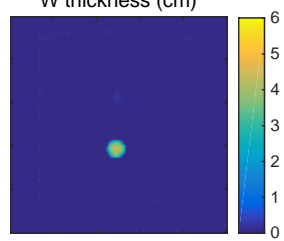

X-ray est. Steel thickness $(\mathrm{cm})$

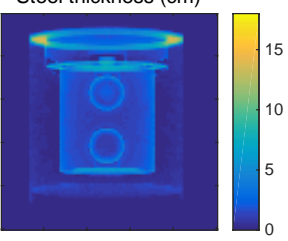

X-ray est.

Poly-pro thickness $(\mathrm{cm})$

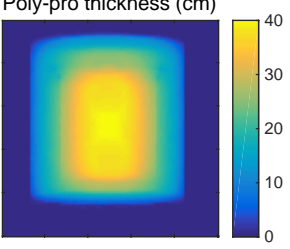

X-ray \& neutron est.

Steel thickness $(\mathrm{cm})$

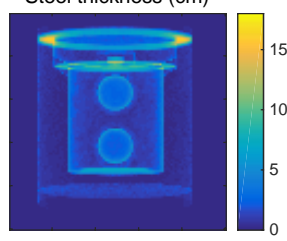

$\mathrm{X}$-ray \& neutron est.

Poly-pro thickness $(\mathrm{cm})$

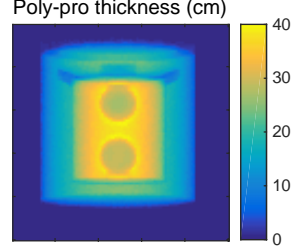

Figure 9. Material estimates when tungsten is added to the material set. The material set is $\{\mathrm{Pu}, \mathrm{W}$, steel, poly-pro $\}$. Note that the discrimination of plutonium and tungsten is practically impossible with only X-ray (left), especially in the presence of image noise. In contrast, the addition of neutron radiography (right) increases material accuracy considerably. 
Table 1: Inspection sets chosen based on the maximum Cramér-Rao lower bound SNR for plutonium, a measure of the ability of the given set to accurately determine the thickness of plutonium from the data. Notice that the optimal sets change based on the chosen material set, seen in the difference when a fourth material (tungsten) is added in the right-most column.

\begin{tabular}{c|c|c} 
Type & $\begin{array}{c}\{\text { Pu, steel, poly-pro }\} \\
\text { Inspection set }\end{array}$ & $\begin{array}{c}\{\text { Pu, W, steel, poly-pro }\} \\
\text { Inspection set }\end{array}$ \\
\hline X-ray & $2,3,7,9 \mathrm{MeV}$ & $4,5,8,9 \mathrm{MeV}$ \\
X-ray, DD or DT & $5,6,9 \mathrm{MeV}, \mathrm{DD}$ or DT & $3,7,9 \mathrm{MeV}, \mathrm{DD}$ or DT \\
X-ray, DD \& DT & $6,9 \mathrm{MeV}, \mathrm{DD}, \mathrm{DT}$ & $5,9 \mathrm{MeV}, \mathrm{DD}, \mathrm{DT}$
\end{tabular}


Table 2: The goodness-of-fit (as RMSE) of results from the algorithm for the best inspection sets from each group based on the Cramér-Rao lower bound SNR. The values shown are the experimental mean and standard deviation of the results from 12 independent noisy data generations. The $\mathrm{RMSE}_{P u}$ is calculated only for the area directly around the projection of the plutonium spheres and the $\mathrm{RMSE}_{n o t} P u$ is calculated for the remaining area of the image.

\begin{tabular}{c|c|c|c} 
Inspection set & $\mathbf{R M S E}_{P u}(\mathbf{c m})$ & $\mathbf{R M S E}_{\text {not } P u}(\mathbf{c m})$ & $\mathbf{P u} \mathbf{E r r}_{\text {mass }}(\mathbf{\%})$ \\
\hline $2,3,7,9 \mathrm{MeV}$ & $0.113 \pm 0.011$ & $0.037 \pm 0.002$ & $0.9 \pm 0.4$ \\
$5,6,9 \mathrm{MeV}, \mathrm{DT}$ & $0.045 \pm 0.007$ & $0.054 \pm 0.002$ & $0.45 \pm 0.25$ \\
$6,9 \mathrm{MeV}, \mathrm{DD}, \mathrm{DT}$ & $0.027 \pm 0.001$ & $0.022 \pm 0.001$ & $0.18 \pm 0.08$
\end{tabular}


Table 3: Various levels scattered flux incident on the image plane will cause an increase in the estimated plutonium error, even when a good estimate of the scatter term is also added to the physics model used in the inverse problem. This is likely because the addition of scattered flux reduces the SNR, making accurate material discrimination more difficult.

\begin{tabular}{c|ccc} 
& \multicolumn{3}{|c}{$\operatorname{RMSE}_{P u}(\mathbf{c m})$} \\
Inspection set & $1 \%$ scatter & $10 \%$ scatter & $100 \%$ scatter \\
\hline $2,3,7,9 \mathrm{MeV}$ & $0.12 \pm 0.02$ & $0.21 \pm 0.01$ & $0.45 \pm 0.08$ \\
$5,6,9 \mathrm{MeV}, \mathrm{DT}$ & $0.07 \pm 0.01$ & $0.12 \pm 0.01$ & $0.67 \pm 0.01$
\end{tabular}

